\title{
Meta-analysis of transcriptome data on oxidative stress response in Saccharomyces cerevisiae cells underlines regulation of carbon, redox and glutathione metabolism
}

\author{
Ezgi Tanıl $(\mathbb{D}$, Burcu Şirin $(\mathbb{D}$, Emrah Nikerel* (10)
}

Yeditepe University, Faculty of Engineering, Department of Genetics and Bioengineering, 34755 Ataşehir, Istanbul, Turkey.

\section{Article History}

Received 05 April 2020

Accepted 20 May 2020

First Online 27 May 2020

\section{*Corresponding Author \\ Tel: +90 (216) 5780619 \\ E-mail: \\ emrah.nikerel@yeditepe.edu.tr}

\section{Keywords}

Oxidative stress

Rank aggregation

Saccharomyces cerevisiae

Meta-analysis

Differential gene expression

\begin{abstract}
Environmental stress adversely affects living systems within medical as well as industrial context, causing either diseases or resulting in e.g. underperforming production processes. In particular oxidative stress in industrial biotechnology context, manifested as the imbalance in generation of reactive oxygen species and antioxidant capacity causes yield losses both in growth and production in baker's yeast. Oxidative stress response studies for Saccharomyces cerevisiae at transcriptome level are using either direct induction methods such as treatment with peroxides or indirect induction methods such as treatment with drugs or toxins. To extract common response mechanisms integrating all conditions is of high value. To this end, this study collects, processes and aggregates published transcriptome data from studies that examined the response using both direct and indirect oxidative stress induction methods. Interestingly, carbon metabolism, oxidation reduction processes and glutathione metabolic process were found to be the common mechanisms involved in oxidative stress response. However, ion homeostasis and hexose transport mechanisms have been shown to be affected from direct induction using peroxides. This result illustrates bioinformatics analysis for large, aggregated transcriptome datasets, as a steppingstone for finding common features and further metabolic engineering targets were developed.
\end{abstract}

\section{Introduction}

Oxidative stress is ubiquitous in living systems from microorganisms to plants and mammalian systems, manifested as the imbalance between the generation of reactive oxygen species (ROS) and the capacity of the biological system to detoxify these intermediates (Inzé \& Van Montagu, 1995; Sies, 2000; Storz \& Imlayt, 1999). The excess free radicals result in damage to fatty tissue, DNA, and proteins causing in turn loss of function, yield or life quality. Coupled with redox imbalance, oxidative stress typically induces system-wide response, involving several transcripts, proteins and biochemical reactions, spanning several omic-layers.

Cells develop complex responses and protection mechanisms to maintain intracellular redox balances against oxidative stress. Responses to oxidative stress are usually on cell survival or cell death mechanisms. Cell survival mechanisms involves antioxidant enzyme mechanisms (SOD, catalase, peroxidase reactions), metabolic products (glutathione, used in almost all eukaryotes) (Poljak et al., 2003), upregulation of various transcription factors (Yap1p, Skn7p, Msn2/4p (Diezmann, 2014)). Besides, necrosis, apoptosis and autophagy are the cell death mechanisms against oxidative stress. These programmed cell death responses of the cell shed light on neurodegenerative diseases and cancer studies (Figure 1) (Farrugia \& Balzan, 2012; Harding et al., 2003; Luo et al., 2017).

Bakers yeast, Saccharomyces cerevisiae, has long been used for centuries not only for making bread or various drinks, but also as food supplement, feed additive (Newbold et al., 1996) as well as for production of bioplastics and other biomaterials (Breuer et al., 2002), biofuels (Cardona et al., 2009) and within medical context (Borresen et al., 2012). As such, it is considered 


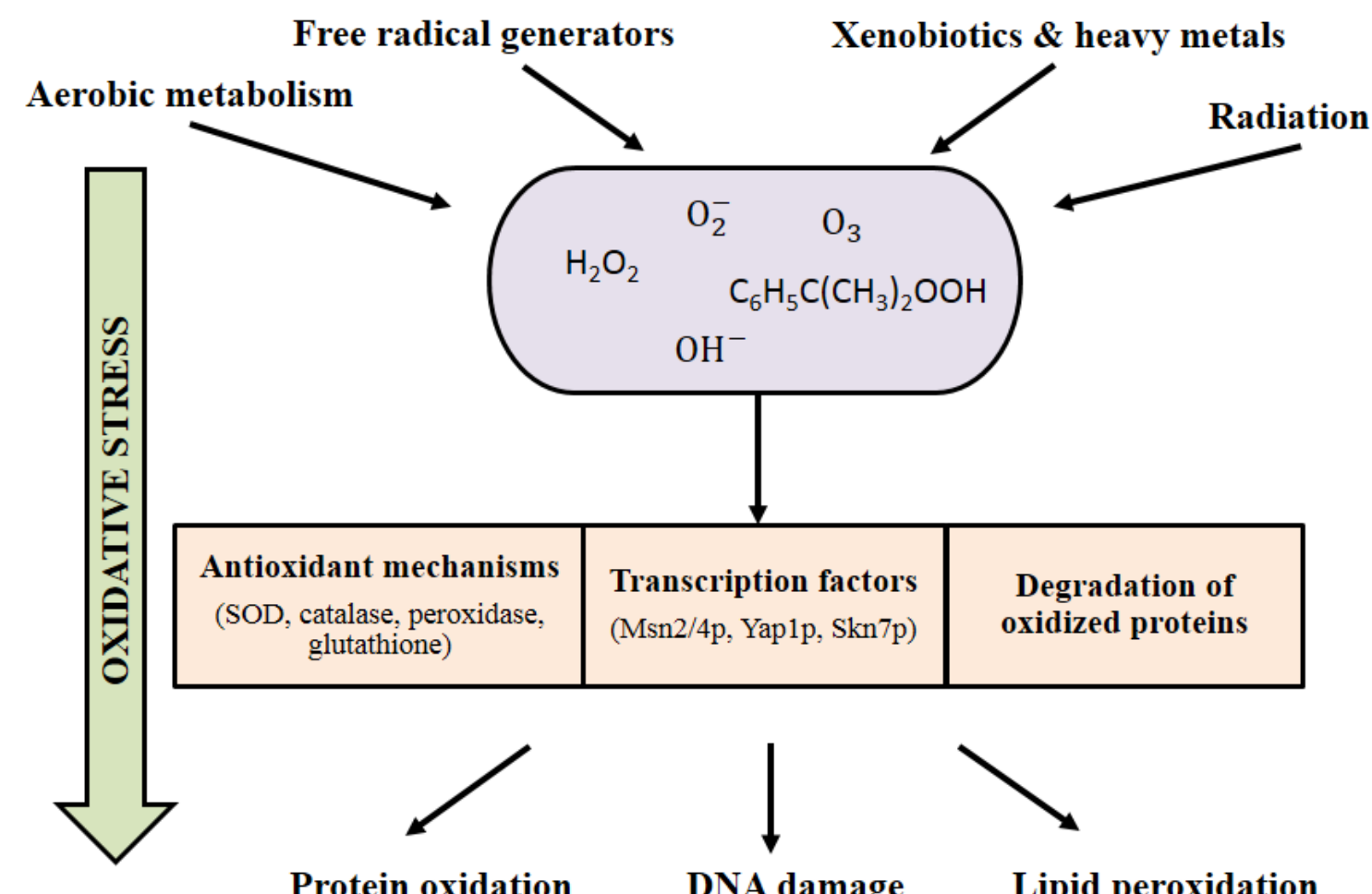

Figure1. A general overview for causes and possible consequences of oxidative stress.

as a model organism for eukaryotic systems as well as an important workhorse for industrial biotechnology (Giaever et al., 2002; Hartwell, 1974; Lee et al., 2002; Li et al., 2004; Mager \& Winderickx, 2005; Nevoigt, 2008; Oliver, 1996; Petranovic \& Vemuri, 2009). The availability of genome sequence (Wei et al., 2007) as well as various molecular biology tools (Mclsaac et al., 2014) renders yeast central not only to understand how cellular machinery works but also for further metabolic engineering studies.

Yeasts are exposed to various stresses (heat, $\mathrm{pH}$, nutrient limitation, osmotic, oxidative) in the industrial context and/or changing environmental conditions. Oxidative stress is an inevitable part of the aerobic life cycles of yeasts. The oxidative stress response in yeast has been evaluated with different perspectives of finding toxicity level of stress agents (Flattery-O'Brien et al., 1993; Jamieson, 1992), identifying the cellular mechanisms (Davies et al., 1995; Gasch et al., 2000), determining stress related genes with mutant strains (Grant et al., 1996, 1998; Okada et al., 2014) and understanding the main mechanisms (Cyrne et al., 2003; Godon et al., 1998; Gopalacharyulu et al., 2009; Matallana \& Aranda, 2017; Peláez-Soto et al., 2020; Piedrafita et al., 2015; Ralser et al., 2007; Yoshimoto et al., 2019). Stress responses have also been studied for living systems for various taxa, ranging from bacteria (Christodoulou et al., 2018; Zhai et al., 2020) to plant cells (Sipari et al., 2020) and other higher eukaryotes (Yu et al., 2020).

In particular, there are several studies focusing on transcriptomic (TX) response. Many studies that investigate transcription factors such as Yap1, Skn7 and
Msn2/4 that regulates oxidative stress response (Carmel-Harel et al., 2001; Livas et al., 2011; Ma \& Liu, 2010; Ouyang et al., 2011; Sha et al., 2013), antioxidant defense mechanisms such as catalases, SOD, thioredoxins and other cellular responses such as cell death and apoptosis following oxidative stress can be found in the literature. However these data obtained from different analysis platforms, oxidative stress inducers and many other experimental and analysis techniques (Cheng et al., 2018; Farrugia et al., 2019; Rodriguez-Colman et al., 2010). Although these studies provide useful information, they cannot be compared to other results of experiments, yet aggregation is of great interest to distill common features of stress response in yeast cells.

A significant challenge in analyzing large scale omic datasets compiled from different sources is to extract key information from experiments performed individually with various agenda, using different techniques or agents, by different labs and/or technicians. In particular for oxidative stress, different labs used various agents $\left(\mathrm{H}_{2} \mathrm{O}_{2}, \mathrm{CHP}\right)$ to induce the stress, alternative techniques (cDNA microarray, oligonucleotide microarray, qPCR, RNA-Seq), alternative experimental design (time-course, static, comparison with a selected mutant) and various strains of Saccharomyces cereviasiae under different growth conditions. Even the purpose of each experiments ranges from determination of toxic/lethal effect to finding resistance genes to stress. A promising approach to analyze omic-dataset from different backgrounds is to perform so-called, meta-analysis of such data. In its essence, meta-analysis aims to aggregate available data 
or information and find key features underlying the system of interest. The approach has been used within various context including oncogenetics (Bhasin et al., 2016), biomarker for plant biotechnology (Zimmermann et al., 2008), stem cell research (Assou et al., 2007) finding stress responsive pathways (Panahi et al., 2019) with even dedicated databases (Hruz et al., 2008).

Rank aggregation is one proposed method for meta-analysis e.g. to combine TX data obtained from different sources (Wald et al., 2012a, 2012b), since the results of high-throughput genomics experiments contain significant amount of noise, and thus reliability of the results should be supported by combining evidences from different experiments or platforms. Therefore, rank aggregation methods provide less noisy results by combining several preference lists. Different approaches proposed in the literature and Robust Rank Aggregation is one of the distribution based approaches which assumes a null probabilistic model to compare each ranked list (Li et al., 2019). The approach consists of focusing on the ranks of genes from individual transcriptome studies, rather than the expression value itself and aggregating list of ranks, assuming betadistribution for the underlying population of lists and scores each element of the combined list with a corrected $p$-value. These corrected $p$-values are sorted to obtain final aggregated ranks. (Kolde et al., 2012; Li et al., 2019)

The advent of high-throughput omic technologies generated ever-growing immense amount of data, which in turn, remains largely under-used and underinterpreted. Making more of the available-omic data is the main approach of our work. The aim of this study is therefore to collect, analyze, aggregate and perform functional analysis of available transcriptome data from response of yeast cells to various oxidative stresses, within the scope of further understanding the effect of this stress on Saccharomyces cerevisiae cells and finding common features among different experiments using bioinformatics tools. In doing so, microarray datasets from NCBI database is collected, inspected for differential expression and compiled a large dataset containing differentially expressed genes in each experiment. Upon rank aggregation and downstream functional analysis, the implications within industrial biotechnology is discussed.

\section{Materials and Methods}

\section{Data Acquisition and Differential Expression Analysis}

All data used in this study has been compiled from public sources. Overall workflow to obtain the transcriptome dataset is presented in Figure 2, including the number of datasets in each step. NCBI Gene Expression Omnibus (GEO) database (https://www.ncbi.nlm.nih.gov/geo/) was searched for the term "oxidative stress" and further filtered for "Saccharomyces cerevisiae".
In this paper, we focused on microarray data, due to standardized experimental and analytical protocols and focused experimental context. In particular, the microarray results from the experiments measuring the cellular response against treatments with oxidants such as peroxides (e.g. $\mathrm{H}_{2} \mathrm{O}_{2}, \mathrm{CHP}$ ), drugs (e.g. Epolauridine) and toxins (e.g. citrinin), directly causing oxidative stress. The list of datasets for the final meta-analysis is given in Table 1 . For samples with replicates (23 of 34 samples), from documented commercial microarray platforms, Differentially Expressed Gene (DEG) analysis was performed using $\mathrm{R}$ limma package (two dye, dye swap and time course designs are also included) and the results were filtered for $p<0.1$ and log fold change, $\log F C>1$. For the samples without replicates, the published logFC was used with the same criteria.

Table 1. Datasets used for meta-analysis.

\begin{tabular}{ll}
\hline Stress Inducer & Accession IDs \\
\hline & GSE45370, GSE58992, \\
& GSE135546, GSE79037, \\
& GSE63030, GSE16346, \\
$\mathrm{H}_{2} \mathrm{O}_{2}$ & GSE26829, GSE55081, \\
& GSE12220, GSE15936, \\
& GSE3406, GSE2977 \\
Linoleic acid hydroperoxide & GSE18334, GSE47820, \\
(LoaOOH) & GSE54951, \\
CHP & GSE7645, GSE26169 \\
Oxygen concentration & GSE22832 \\
Transition from anaerobic to aerobic & GSE7140 \\
TiO ${ }_{2}+$ UV & GSE99660 \\
Mycotoxins: citrinin (CIT) and & GSE84187 \\
ochratoxin A (OTA) & GSE6185 \\
Pyocyanin & GSE101749 \\
Eupolauridine-9591 (E9591) & GSE3683 \\
Menadione & GSE5608 \\
Celastrol & GSE6067, GSE6068 \\
Arsenite & GSE18411 \\
Zinc & GSE24712 \\
\hline & \\
\hline &
\end{tabular}

For time-course experiments (11 of 34), the last time point or, 30/60/90-minute time point (if available) was compared to control taken prior to treatment. For each sample, the absolute values of logFC upon filtering were ranked in increasing order and these ranks were normalized with respect to maximum rank in the corresponding sample. Finally, normalized ranks for each gene among all of the samples were merged to generate the rank matrix that contains normalized ranks of 6039 ORF's in 34 samples. 


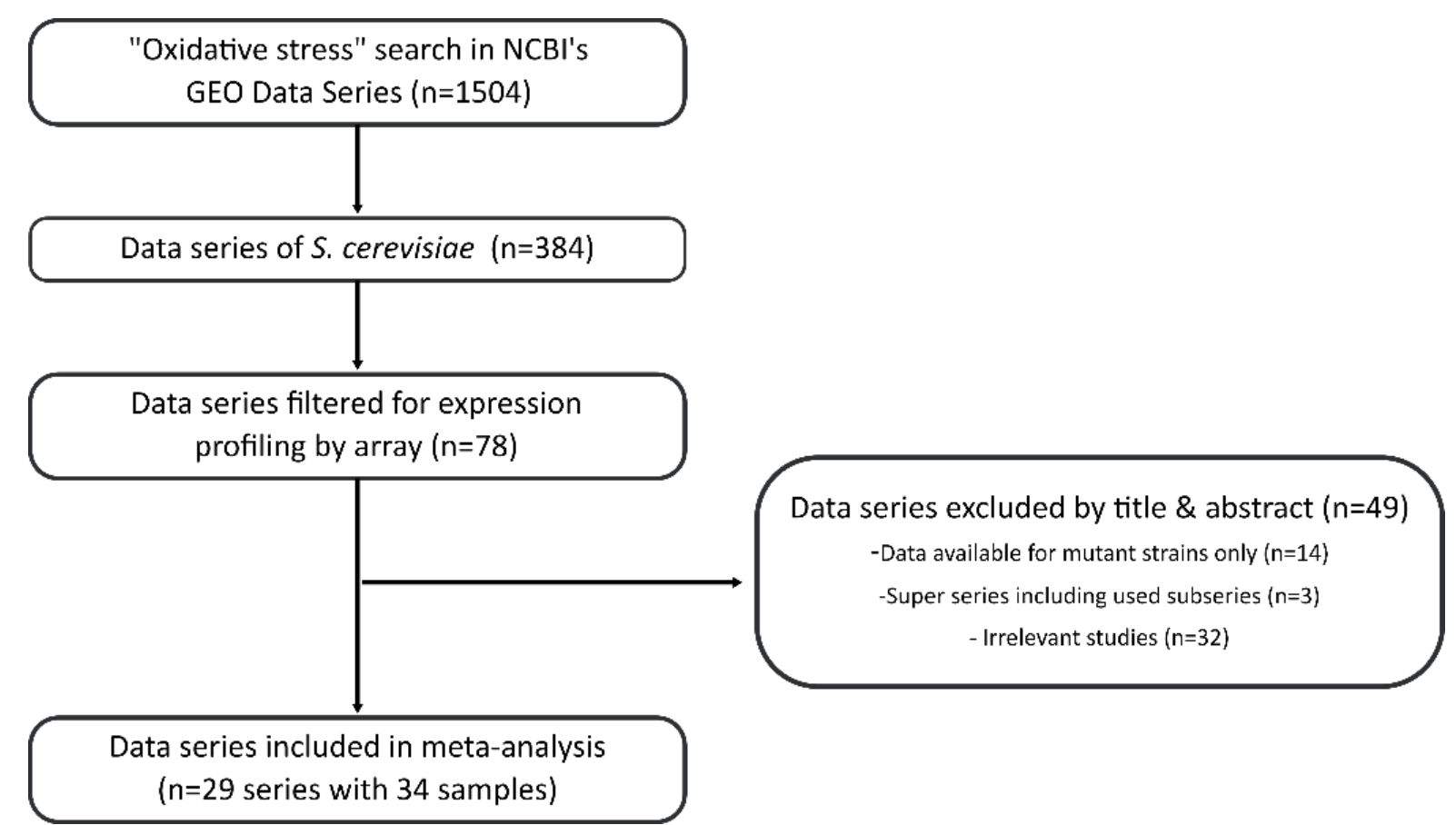

Figure 2. Transcriptome data acquisition workflow to compile the data used in this study.

\section{Aggregation of transcriptome data}

The aggregation of transcriptome data is performed using Robust Rank Aggregation algorithm and the R package RobustRankAggreg (Kolde et al., 2012). In case the transcript level for a specific probe, the corresponding entry to the rank matrix was replaced with maximum normalized rank value, namely 1 . The rank matrix was aggregated using aggregateRanks function, which calculates $\rho$-scores for each transcript and sorts them in increasing order.

\section{Functional analysis of differentially expressed genes in aggregated list}

\section{GO and KEGG Enrichment}

For the ORF's that are overrepresented in all datasets are determined based on their $\rho$-scores, and a cutoff-score of 0.1 . For overrepresented genes ( $\rho<$ 0.1 ), gene set enrichment analysis based on Gene Onthology (GO) and KEGG pathways were performed using $\mathrm{R}$ packages clusterProfiler and org.Sc.sgd.db Bioconductor genome wide annotation for yeast, and DAVID Gene Functional Annotation and Classification Tools (https://david.ncifcrf.gov/home.jsp) and AmiGO database (Carbon et al., 2009). For GO annotation for all GO ontologies, molecular function, bioprocess and cellular component, were performed using enrichGO function using Ensembl ID's as key types, false discovery rate as $p$ value adjustment method and $p$-value cutoff was set to 0.1 (same in KEGG enrichment). The KEGG annotation was performed similarly using NCBI Gene ID's as keys using enrichKEGG function.

\section{Results and Discussion}

This study focuses on finding common and significant gene sets, differentially expressed upon oxidative stress, using meta-analysis of transcriptome data, collected from public databases. The robust rank aggregation method used here allows to overrepresented genes. It should be noted that the final set of genes may or may not be the most differentially expressed all together in a single dataset, rather, the final set of genes are differentially expressed in most (evaluated with statistically significance) of the datasets considered. The overall workflow for selection of microarray transcriptome datasets is given in Figure 2. The selected final datasets with 34 samples include direct or indirect induction of oxidative stress response on wild-type strains, while studies including mutant strains, investigating unconventional conditions such as radioactive materials or freezing were not considered. This way, more focused experimental context was obtained.

DEG analysis was de novo performed for series containing replicates or series where raw data is available in .CEL, .txt or .gpr formats. The ranking of transcripts was performed based on absolute logFC, so up- or downregulation was not separately investigated in aggregated list. Normalization of the ranks considering the number of the differentially expressed genes allows lowering the effect of unreliable DEG lists. In case, the raw data or replicates are not available in a specific dataset, statistical significance of the differential expression cannot be known, yet, published information on logFC is used to obtain statistically significant aggregated ranks. 
Upon DEG analysis for each sample, the normalized rank lists from each sample were merged to obtain $10715 \times 34$ rank matrix (10715 ORF's and 34 samples) containing normalized rank list, one for each sample. Following the work of Kolde 2012, p-values for beta distribution were calculated (and corrected for possible false positives due to multiple hypothesis testing) for each transcript. Finally, $83 \mathrm{ORF}^{\prime}$ s have $\rho$-score less than 1 and 42 of these were considered to be overrepresented in all data set ( $\rho<0.1$, Table 2).

Oxidative stress might be induced directly by perturbing the culture with e.g. a hyperoxide or indirectly using excess aeration. To assess whether the stress induction method delivers different set of genes or not, a subset of $10 \mathrm{DEG}$ lists that contains only direct induction methods by $\mathrm{H}_{2} \mathrm{O}_{2}$, LoaOOH and $\mathrm{CHP}$, were aggregated. Comparison of the results were shown in Figure 3. In this case, 23 of the 10715 ORF's had $\rho$-score less than 0.1. Interestingly, the 23 ORFs were not a subset of the initial list 43 ORF's obtained from the dataset with 34 samples, though several genes are shared between these two lists (Figure 3).

Table 2. $\rho$-scores of significantly differentially expressed genes in analyses containing all samples and only samples with direct induction of oxidative stress.

\begin{tabular}{|c|c|c|c|}
\hline ORF & Description & $\begin{array}{c}\rho \text { Score } \\
\text { (All Samples) }\end{array}$ & $\begin{array}{c}\rho \text { Score } \\
\text { (Direct induction) }\end{array}$ \\
\hline YPL171C & NADPH dehydrogenase & $2.80 \mathrm{E}-09$ & $2.26 \mathrm{E}-06$ \\
\hline YKL071W & $\mathrm{NADH}$-dependent aldehyde reductase & $1.14 \mathrm{E}-08$ & 0.001887 \\
\hline YKL086W & sulfiredoxin & $6.72 \mathrm{E}-08$ & $6.57 \mathrm{E}-06$ \\
\hline YDR453C & thioredoxin peroxidase TSA2 & 2.07E-06 & $9.28 \mathrm{E}-05$ \\
\hline YOL052C-A & Ddr2p, Multi-stress response protein & $1.54 \mathrm{E}-05$ & 0.017453 \\
\hline YBR072W & chaperone protein HSP26 & $3.25 \mathrm{E}-05$ & 0.084638 \\
\hline YCR021C & Hsp30p & 4.89E-05 & 0.043349 \\
\hline YMR090W & Putative protein of unknown function & $6.28 \mathrm{E}-05$ & - \\
\hline YLL060C & glutathione transferase GTT2 & $2.54 \mathrm{E}-04$ & 0.006030 \\
\hline YOR382W & Fit2p & $2.83 \mathrm{E}-04$ & 0.003935 \\
\hline YHR048W & Yhk8p & 3.05E-04 & $1.12 \mathrm{E}-04$ \\
\hline YER103W & Hsp70 family chaperone SSA4 & 4.07E-04 & - \\
\hline YHR087W & Rtc3p & 0.000776 & 0.044299 \\
\hline YCL026C-A & type II nitroreductase & 0.001375 & 0.003311 \\
\hline YLR327C & Tma10p & 0.001497 & - \\
\hline YGR248W & 6-phosphogluconolactonase SOL4 & 0.002262 & 0.071480 \\
\hline YML131W & Protein of unknown function & 0.002967 & - \\
\hline YKR076W & S-glutathionyl-(chloro)hydroquinone reductase & 0.003412 & 0.035562 \\
\hline YDL243C & putative aryl-alcohol dehydrogenase & 0.005133 & - \\
\hline YGR088W & catalase $\mathrm{T}$ & 0.005402 & \\
\hline YFL053W & dihydroxyacetone kinase & 0.006326 & 0.024013 \\
\hline YBR244W & glutathione peroxidase GPX2 & 0.007829 & 0.015116 \\
\hline YGR224W & azole transporter & 0.007841 & - \\
\hline YER067W & Rgi1p & 0.008506 & - \\
\hline YFL014W & lipid-binding protein HSP12 & 0.012983 & - \\
\hline YMR173W & DNA damage-responsive protein 48 & 0.019120 & 0.069446 \\
\hline YFL056C & Putative aryl-alcohol dehydrogenase & 0.023097 & - \\
\hline YDR171W & heat shock protein HSP42 & 0.023735 & - \\
\hline YNL194C & Integral membrane protein & 0.026158 & - \\
\hline YDR256C & catalase A & 0.028125 & 0.093993 \\
\hline YLR205C & $\mathrm{Hm} \times 1 \mathrm{p}$ & 0.031368 & 0.011914 \\
\hline YDL204W & $\operatorname{Rtn} 2 p$ & 0.035241 & - \\
\hline YGR008C & ATPase-stabilizing factor family protein & 0.036289 & - \\
\hline
\end{tabular}


Table 2. (continued)

\begin{tabular}{llcc}
\hline YML128C & Msc1p & 0.038967 & - \\
YLR346C & Cis1p & 0.045650 & - \\
YHR139C & Sps100p & 0.046043 & 0.054276 \\
YDR533C & glutathione-independent methylglyoxalase & 0.050285 & - \\
YOL151W & methylglyoxal reductase (NADPH-dependent) GRE2 & 0.040635 \\
YGR035C & Putative protein of unknown function, & 0.056597 & - \\
YDL246C & L-iditol 2-dehydrogenase SOR2 & 0.065623 & - \\
YKL070W & uncharacterized protein & 0.071729 & - \\
YLR136C & Tis11p & 0.095622 & 0.037149 \\
YFR053C & hexokinase 1 & - & 0.050526 \\
YBR047W & Fmp23p & - & 0.054616 \\
YMR011W & hexose transporter HXT2 & - & 0.072490 \\
YJR005C-A & Lso1p & - & 0.097781 \\
YPR030W & Csr2p & - & 0.098063
\end{tabular}

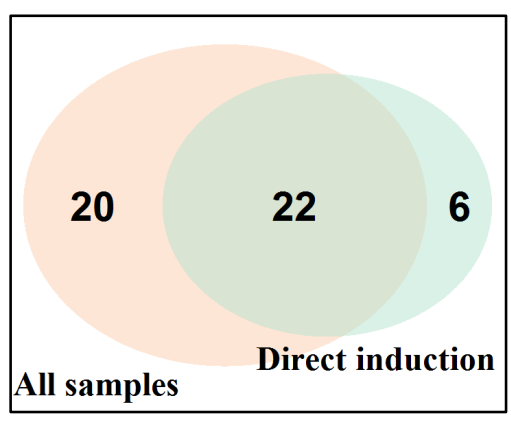

Figure 3. Venn diagram of the number of ORF's that are differentially expressed in aggregated list of all samples and the samples that were obtained from direct induction experiments with replicates.

To gain further insights on the functions of the aggregated list of genes, gene set enrichment analysis based on GO-terms was performed. Enriched GO-terms in the resulting gene set in terms of biological process and molecular function are presented in Figure $4 \mathrm{a}$ and 4b. Most overrepresented GO-terms are oxidative stress (30\% of the genes), cellular response to chemical stimulus ( $36 \%$ of the genes) and oxidation-reduction process ( $40 \%$ of the genes) for both "all samples" and "direct oxidative stress method samples" lists.

Interestingly, 2 genes in both lists are annotated to be involved in glutathione metabolic process. Differences also exists in enriched GO-terms, in both lists. In direct induction list, 5 (out of 28) genes are annotated in chemical homeostasis and 2 genes are annotated in fructose/glucose transmembrane transport. In molecular function, similar GO-term landscape is observed. In "all samples list", 14 genes are annotated in oxidoreductase activity, 2 genes in glutathione reductase activity. Lastly for cellular component, $60 \%$ of the ORFs were located in cytoplasm, $15 \%$ in cell periphery and $18 \%$ in plasma membrane. Further manual annotation using Yeast Genome (https://www.yeastgenome.org) website resulted in 16 manually curated GO-cellular component annotation, 8 in cytoplasm and 5 in nucleus.

To get more insight into gene sets, we further focused on the set of genes in two GO terms, (i) glutathione metabolic process (GO:0006749) and response to oxidative stress (GO:0006979), containing 19 ORF's and 125 ORF's respectively according to AmiGO. For those ORFs, the $\rho$-scores were plotted, showing the count of the samples that the specific ORF was differentially expressed (Figure 5). For glutathione metabolic process, 18 of the genes were found to be differentially expressed in more than two samples, but only two of them YKR076W (ECM4, differentially expressed in 18 samples) and YLL060C (GTT2, differentially expressed in 20 samples) were found to be significantly different in all samples. For response to oxidative stress, twelve of 125 genes were found to be significantly differentially expressed in all samples (average number of samples that these genes were found to be differentially expressed 16).

Upon aggregation, 28 genes were found to be significantly overrepresented in the lists of differential expressed genes from individual experiments. These genes are categorized as responsible for antioxidant capacity, oxidoreductase activity (acting on peroxide as acceptor, acting on a sulfur group of donors, and acting on $\mathrm{NAD}(\mathrm{P}) \mathrm{H})$, peroxidase mechanisms, glutathione transferase activity, peroxiredoxin mechanisms, heme binding and tetrapyrrole binding activities. The majority of the above stated functions are related to the electron transfers or mechanisms for reducing the oxidative stress.

These genes are further inspected in KEGG database to further probe their biological context. Significant hits are observed at longevity regulating pathway, carbon metabolism, tryptophan metabolism, fructose and mannose metabolism, protein processing in endoplasmic reticulum, glyoxylate and dicarboxylate metabolism, MAPK signaling pathway - yeast, 

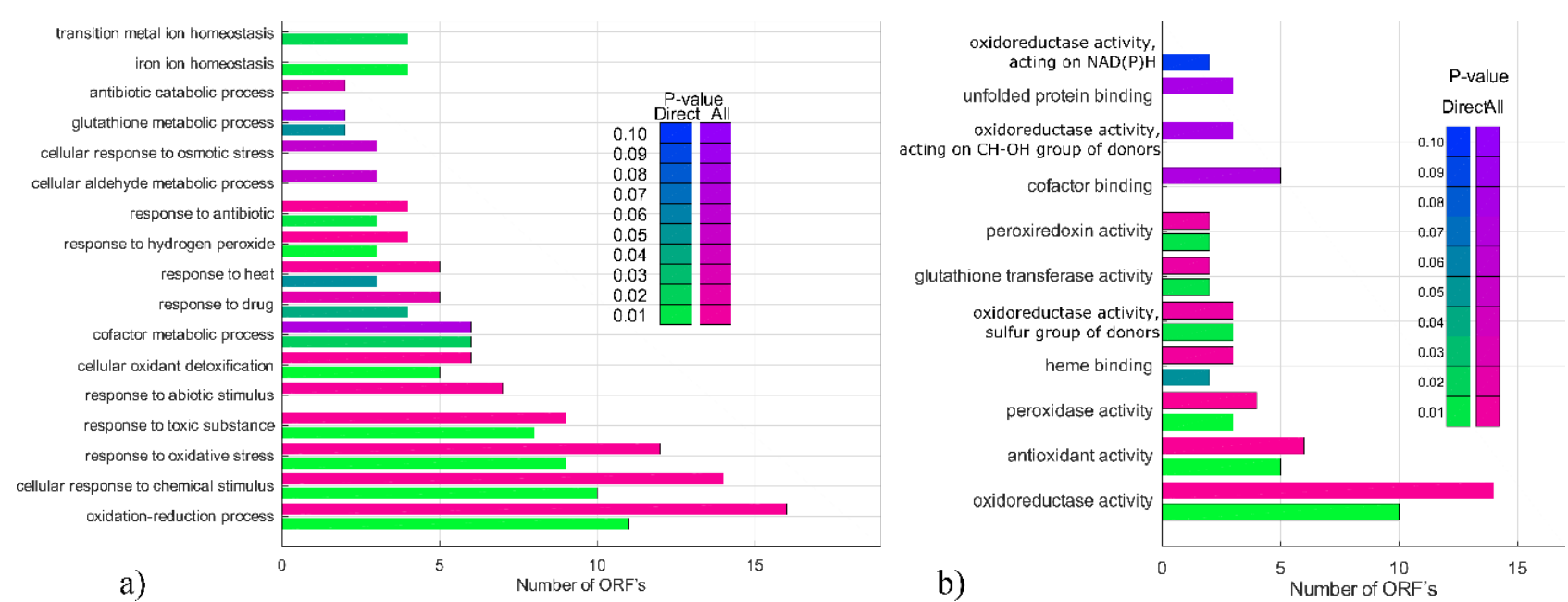

Figure 4. GO enrichment results for all list of samples which direct induction applied (a) GO-Bioprocess (b) GO-Molecular function.

peroxisome, pyruvate metabolism, pentose and glucuronate interconversions and propanoate metabolism. Interestingly, when the focused dataset (direct oxidative stress), carbon metabolism and fructose and mannose metabolism are the main hits metabolic pathways, especially with hydrogen peroxideinduced oxidative stress, aiming at increasing NADPH, an important cofactor in the oxidation reduction mechanisms (glutaredoxins, thioredoxins). Also, trehalose synthesis and synthesis of three enzymes

(glucose-6-phosphate dehydrogenase, transketolases and transaldolase) of pentose phosphate pathways induced (Godon et al., 1998) overall increasing NADPH levels.

Next to cofactor balances, oxidative stress also induces antioxidant defense mechanisms, a protein degradation pathway. Glutathione plays a role against oxidative stress in yeast both as a metabolite in nonenzymatic mechanisms and as a cofactor in enzymatic mechanisms (glutathione peroxidase, glutathione

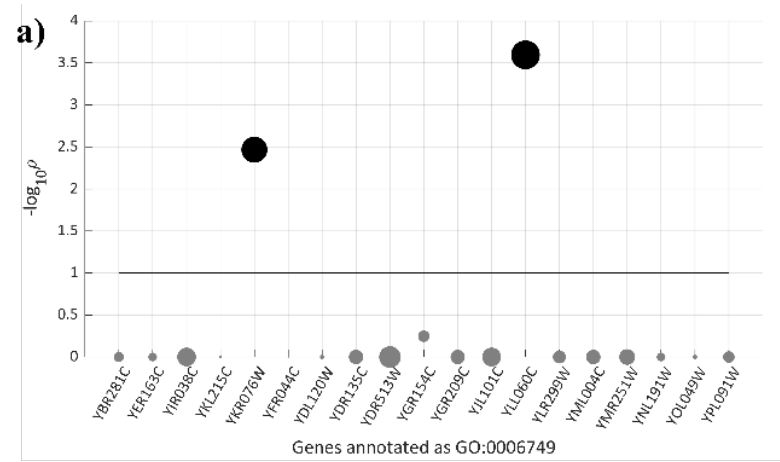

b)

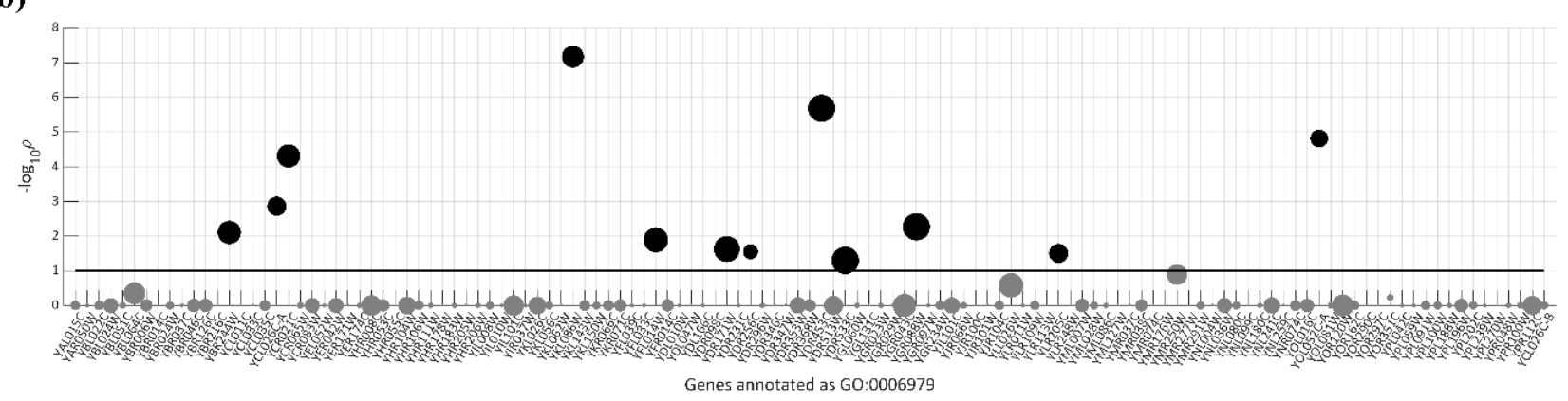

Figure 1. (a) Rank scores of ORF's involved in glutathione metabolic process. ORF's that differentially expressed were shown with black dots, line represents significance level $(r h o=0.1)$ and marker sizes refer to number of samples that these ORF's were found to be differentially expressed. (b) Rank scores of ORF's involved in response to oxidative stress. ORF's that differentially expressed were shown with black dots, line represents significance level $(\rho=0.1)$ and marker sizes refer to number of samples that these ORF's were found to be differentially expressed. 
reductase (Douglas, 1987, Meister, 1988). The protein degradation via $\mathrm{H}_{2} \mathrm{O}_{2}$-induced oxidative stress causing oxidation of major proteins (pyruvate decarboxylase, fatty acid synthase, and glyceraldehyde-3-phosphate dehydrogenase (Tdh) are also separately reported in literature (Cabiscol et al., 2000).

This work combines several datasets related to oxidative stress in yeast. It should be noted that a typical extension is a detailed analysis that would require careful grouping taking into account each stressor as well as corresponding (known) defense or response mechanisms and intracellular indicators (protein carbonylation, Thiobarbituric Acid Reactive Substances (TBARS) etc.). In selecting the datasets (Figure 2), we adopt a top-down approach and care is taken not to "over-slice" the data by too-detailed-subgrouping, allowing not to lose general features.

In performing meta-analysis studies, one expected pitfall is on dosage experiments, where the level of stressor triggers different or additional response/defense mechanisms. In that case, the ranking of a selected transcript will like change. However, considering nearly 6000 all genes in yeast, it would be highly unlikely to find such a finely tuned transcript that would be common key to all experiments due to robustness of metabolism. One specific transcript will be of key importance of that specific, isolated experiment, and is expected to be diluted over other experiments. In contrast, the transcripts in first couple of rows in Table 2 are found nearly always among top 20 differentially expressed genes.

\section{Conclusion}

Identification of key processes as a response to environmental stress is one of the key problems in biology, not only for our fundamental understanding but also to rational design of improved industrial strains and processes. Focusing on oxidative stress response, robust rank aggregation was performed to extract useful information from various experiments as individual experiments uses different techniques, have different agenda and are performed by different labs. This work strived for finding metabolic engineering targets for improved yields and productivities of industrially important microorganisms. Analysis underlined that key processes center around carbon, redox and glutathione metabolism and pointed interplay among those. In conclusion, this study illustrates bioinformatics analysis on capturing common features in a large, aggregated datasets and points to key common features and further metabolic engineering targets. Further investigation on distribution and possible redirection of metabolic fluxes allow further pinpointing key reactions.

\section{Acknowledgements}

Financial support for Burcu Şirin through TÜBiTAK 2211C Program (1649B03) and Ezgi Tanıl from Yeditepe
University Research Fund is gratefully acknowledged.

\section{References}

Assou, S., Le Carrour, T., Tondeur, S., Ström, S., Gabelle, A., Marty, S., Nadal, L., Pandesco, V., Reme, T. Hugnot, J. P., Gasca, S., Hovatta, O., Hamamah, S., Klein, B., \& De Vos, J. (2007). A meta-analysis of human embryonic stem cells transcriptome integrated into a web-based expression atlas. Stem cells, 25(4), 961-973.

Bhasin, M. K., Ndebele, K., Bucur, O., Yee, E. U., Otu, H. H., Plati, J., Bullock, A., Gu, X., Castan, E., Zhang, P., Najarian, R., Muraru, M. S., Miksad, R., Khosravi-Far, R., \& Libermann, T. A. (2016). Meta-analysis of transcriptome data identifies a novel 5-gene pancreatic adenocarcinoma classifier. Oncotarget, 7(17), 23263.

Breuer, U., Terentiev, Y., Kunze, G., \& Babel, W. (2002). Yeasts as producers of polyhydroxyalkanoates: genetic engineering of Saccharomyces cerevisiae. Macromolecular bioscience, 2(8), 380-386.

Borresen, E. C., Henderson, A. J., Kumar, A., Weir, T. L., \& Ryan, E. P. (2012). Fermented foods: patented approaches and formulations for nutritional supplementation and health promotion. Recent patents on food, nutrition \& agriculture, 4(2), 134-140.

Cabiscol, E., Piulats, E., Echave, P., Herrero, E., \& Ros, J. (2000). Oxidative stress promotes specific protein damage in Saccharomyces cerevisiae. Journal of Biological Chemistry, 275(35), 27393-27398.

Carbon, S., Ireland, A., Mungall, C. J., Shu, S., Marshall, B., Lewis, S., the Amigo Hub, \& the Web Presence Working Group. (2009). AmiGO: online access to ontology and annotation data. Bioinformatics, 25(2), 288-289.

Cardona, C. A., Orrego, C. E., \& Paz, I. C. (2009). The Potential for production of bioethanol and bioplastics from potato starch in Colombia. Fruit, Vegetable and Cereal Science and Biotechnology, 3, 102-114.

Carmel-Harel, O., Stearman, R., Gasch, A. P., Botstein, D., Brown, P. O., \& Storz, G. (2001). Role of thioredoxin reductase in the Yap1p-dependent response to oxidative stress in Saccharomyces cerevisiae. Molecular microbiology, 39(3), 595-605.

Cheng, C., Tang, R. Q., Xiong, L., Hector, R. E., Bai, F. W., \& Zhao, X. Q. (2018). Association of improved oxidative stress tolerance and alleviation of glucose repression with superior xylose-utilization capability by a natural isolate of Saccharomyces cerevisiae. Biotechnology for biofuels, 11(1), 28.

Christodoulou, D., Link, H., Fuhrer, T., Kochanowski, K., Gerosa, L., \& Sauer, U. (2018). Reserve flux capacity in the pentose phosphate pathway enables Escherichia coli's rapid response to oxidative stress. Cell systems, 6(5), 569-578.

Cyrne, L., us Martins, L., Fernandes, L., \& Marinho, H. S. (2003). Regulation of antioxidant enzymes gene expression in the yeast Saccharomyces cerevisiae during stationary phase. Free Radical Biology and Medicine, 34(3), 385393.

Davies, J. M., Lowry, C. V., \& Davies, K. J. (1995). Transient adaptation to oxidative stress in yeast. Archives of biochemistry and biophysics, 317(1), 1-6.

Diezmann, S. (2014). Oxidative stress response and adaptation to $\mathrm{H} 2 \mathrm{O} 2$ in the model eukaryote Saccharomyces cerevisiae and its human pathogenic relatives Candida albicans and Candida glabrata. Fungal Biology Reviews, 
28(4), 126-136.

Douglas, K. T. (1987). Mechanism of action of glutathionedependent enzymes. Advances in enzymology and related areas of molecular biology, 59, 103-167.

Farrugia, G., Azzopardi, M., Saliba, C., Grech, G., Gross, A. S., Pistolic, J., Benes, V., Vassallo, N., Borg, J., Madeo, F., Eisenberg, T., \& Balzan, R. (2019). Aspirin impairs acetylcoenzyme $A$ metabolism in redox-compromised yeast cells. Scientific reports, 9(1), 1-13.

Flattery-O'Brien, J., Collinson, L. P., \& Dawes, I. W. (1993). Saccharomyces cerevisiae has an inducible response to menadione which differs from that to hydrogen peroxide. Microbiology, 139(3), 501-507.

Gasch, A. P., Spellman, P. T., Kao, C. M., Carmel-Harel, O., Eisen, M. B., Storz, G., Botstein, D., \& Brown, P. O. (2000). Genomic expression programs in the response of yeast cells to environmental changes. Molecular biology of the cell, 11(12), 4241-4257.

Giaever, G., Chu, A. M., Ni, L., Connelly, C., Riles, L., Véronneau, S., Dow, S., Lucau-Danila, A., Anderson, K., Andre, B., Arkin, A. P., Astromoff, A., Bakkoury, M. E., Bangham, R., Benito, R., Brachat, S., Campanaro, S., Curtiss, M., ... \& Johnston, M. (2002). Functional profiling of the Saccharomyces cerevisiae genome. Nature, 418(6896), 387-391.

Godon, C., Lagniel, G., Lee, J., Buhler, J. M., Kieffer, S., Perrot, M., Boucherie, H., Toledano, M. B., \& Labarre, J. (1998). The $\mathrm{H} 2 \mathrm{O} 2$ stimulon in Saccharomyces cerevisiae. Journal of Biological Chemistry, 273(35), 22480-22489.

Gopalacharyulu, P. V., Velagapudi, V. R., Lindfors, E., Halperin, E., \& Orešič, M. (2009). Dynamic network topology changes in functional modules predict responses to oxidative stress in yeast. Molecular BioSystems, 5(3), 276-287.

Grant, C. M., Maclver, F. H., \& Dawes, I. W. (1996). Glutathione is an essential metabolite required for resistance to oxidative stress in the yeastSaccharomyces cerevisiae. Current genetics, 29(6), 511-515.

Grant, C. M., Perrone, G., \& Dawes, I. W. (1998). Glutathione and catalase provide overlapping defenses for protection against hydrogen peroxide in the yeastsaccharomyces cerevisiae. Biochemical and biophysical research communications, 253(3), 893-898.

Harding, H. P., Zhang, Y., Zeng, H., Novoa, I., Lu, P. D., Calfon, M., Sadri, N., Yun, C., Popko, B., Paules, R., Stojdl, D. F., Bell, J. C., Hettmann, T., Leiden, J. M., \& Ron, D. (2003). An integrated stress response regulates amino acid metabolism and resistance to oxidative stress. Molecular cell, 11(3), 619-633.

Hartwell, L. H. (1974). Saccharomyces cerevisiae cell cycle. Bacteriological reviews, 38(2), 164.

Hruz, T., Laule, O., Szabo, G., Wessendorp, F., Bleuler, S., Oertle, L., Widmayer, P., Gruissem, W., \& Zimmermann, P. (2008). Genevestigator v3: a reference expression database for the meta-analysis of transcriptomes. Advances in bioinformatics. 2008, 420747

Inzé, D., \& Van Montagu, M. (1995). Oxidative stress in plants. Current Opinion in Biotechnology, 6(2), 153-158.

Jamieson, D. J. (1992). Saccharomyces cerevisiae has distinct adaptive responses to both hydrogen peroxide and menadione. Journal of bacteriology, 174(20), 66786681.

Kolde, R., Laur, S., Adler, P., \& Vilo, J. (2012). Robust rank aggregation for gene list integration and meta- analysis. Bioinformatics, 28(4), 573-580.

Lee, T. I., Rinaldi, N. J., Robert, F., Odom, D. T., Bar-Joseph, Z., Gerber, G. K., Hannett, N. M., Harbison, C. T., Thompson, C. M., Simon, I., Zeitlinger, J., Jennings, E.G., Murray, H. L., Gordon, D. B., Ren, B., Wyrick, J. J., Tagne, J. B., Volkert, T. L., Fraenkel, E., Gifford, D. K., \& Young, R. A. (2002). Transcriptional regulatory networks in Saccharomyces cerevisiae. Science, 298(5594), 799-804.

Li, X., Wang, X., \& Xiao, G. (2019). A comparative study of rank aggregation methods for partial and top ranked lists in genomic applications. Briefings in bioinformatics, 20(1), 178-189.

Li, Y., Wei, G., \& Chen, J. (2004). Glutathione: a review on biotechnological production. Applied microbiology and biotechnology, 66(3), 233-242.

Livas, D., Almering, M. J., Daran, J. M., Pronk, J. T., \& Gancedo, J. M. (2011). Transcriptional responses to glucose in Saccharomyces cerevisiae strains lacking a functional protein kinase A. BMC genomics, 12(1), 405.

Luo, H., Chiang, H. H., Louw, M., Susanto, A., \& Chen, D. (2017). Nutrient sensing and the oxidative stress response. Trends in Endocrinology \& Metabolism, 28(6), 449-460.

Ma, M., \& Liu, Z. L. (2010). Comparative transcriptome profiling analyses during the lag phase uncover YAP1, PDR1, PDR3, RPN4, and HSF1 as key regulatory genes in genomic adaptation to the lignocellulose derived inhibitor HMF for Saccharomyces cerevisiae. BMC genomics, 11(1), 660.

Mager, W. H., \& Winderickx, J. (2005). Yeast as a model for medical and medicinal research. Trends in pharmacological sciences, 26(5), 265-273.

Matallana, E., \& Aranda, A. (2017). Biotechnological impact of stress response on wine yeast. Letters in applied microbiology, 64(2), 103-110.

Mclsaac, R. S., Gibney, P. A., Chandran, S. S., Benjamin, K. R., \& Botstein, D. (2014). Synthetic biology tools for programming gene expression without nutritional perturbations in Saccharomyces cerevisiae. Nucleic acids research, 42(6), e48-e48.

Meister, A. (1988). Glutathione metabolism and its selective modification. Journal of biological chemistry, 263(33), 17205-17208.

Nevoigt, E. (2008). Progress in metabolic engineering of Saccharomyces cerevisiae. Microbiol. Mol. Biol. Rev., 72(3), 379-412.

Newbold, C. J., Wallace, R. J., \& McIntosh, F. M. (1996). Mode of action of the yeast Saccharomyces cerevisiae as a feed additive for ruminants. British Journal of Nutrition, 76(2), 249-261.

Okada, N., Ogawa, J., \& Shima, J. (2014). Comprehensive analysis of genes involved in the oxidative stress tolerance using yeast heterozygous deletion collection. FEMS yeast research, 14(3), 425-434.

Oliver, S. G. (1996). From DNA sequence to biological function. Nature, 379(6566), 597-600.

Ouyang, X., Tran, Q. T., Goodwin, S., Wible, R. S., Sutter, C. H., \& Sutter, T. R. (2011). Yap1 activation by $\mathrm{H} 2 \mathrm{O} 2$ or thiolreactive chemicals elicits distinct adaptive gene responses. Free Radical Biology and Medicine, 50(1), 113.

Panahi, B., Dumas, J., \& Hejazi, M. (2019). Integration of cross species RNA-seq Meta-analysis and Machine Learning Models identifies the most important salt stress responsive pathways in microalga Dunaliella. Frontiers in 
genetics, 10, 752.

Peláez-Soto, A., Roig, P., Martínez-Culebras, P. V., FernándezEspinar, M. T., \& Gil, J. V. (2020). Proteomic Analysis of Saccharomyces cerevisiae Response to Oxidative Stress Mediated by Cocoa Polyphenols Extract. Molecules, 25(3), 452.

Petranovic, D., \& Vemuri, G. N. (2009). Impact of yeast systems biology on industrial biotechnology. Journal of Biotechnology, 144(3), 204-211.

Piedrafita, G., Keller, M. A., \& Ralser, M. (2015). The impact of on-enzymatic reactions and enzyme promiscuity on cellular metabolism during (oxidative) stress conditions. Biomolecules, 5(3), 2101-2122.

Poljak, A., Dawes, I. W., Ingelse, B. A., Duncan, M. W., Smythe, G. A., \& Grant, C. M. (2003). Oxidative damage to proteins in yeast cells exposed to adaptive levels of H2O2. Redox report, 8(6), 371-377.

Ralser, M., Wamelink, M. M., Kowald, A., Gerisch, B., Heeren, G., Struys, E. A., Klipp, E., Jakobs, C., Breitenbach, M., Lehrach, H., \& Krobitsch, S. (2007). Dynamic rerouting of the carbohydrate flux is key to counteracting oxidative stress. Journal of Biology, 6(4), 10.

Rodriguez-Colman, M. J., Reverter-Branchat, G., Sorolla, M. A., Tamarit, J., Ros, J., \& Cabiscol, E. (2010). The forkhead transcription factor $\mathrm{Hcm} 1$ promotes mitochondrial biogenesis and stress resistance in yeast. Journal of Biological Chemistry, 285(47), 37092-37101.

Sha, W., Martins, A. M., Laubenbacher, R., Mendes, P., \& Shulaev, V. (2013). The genome-wide early temporal response of Saccharomyces cerevisiae to oxidative stress induced by cumene hydroperoxide. PloS One, 8(9), e74939.

Sies, H. (2000). What is oxidative stress?. In Oxidative stress and vascular disease (pp. 1-8). Springer, Boston, MA.

Sipari, N., Lihavainen, J., Shapiguzov, A., Kangasjärvi, J., \& Keinänen, M. (2020). Primary Metabolite Responses to Oxidative Stress in Early-Senescing and Paraquat Resistant Arabidopsis thaliana rcd1 (Radical-Induced Cell Death1). Frontiers in plant science, 11, 194.
Wald, R., Khoshgoftaar, T. M., \& Dittman, D. (2012a). Mean aggregation versus robust rank aggregation for ensemble gene selection. In 2012 11th International Conference on Machine Learning and Applications (Vol. 1, pp. 63-69). IEEE.

Wald, R., Khoshgoftaar, T. M., Dittman, D., Awada, W., \& Napolitano, A. (2012b). An extensive comparison of feature ranking aggregation techniques in bioinformatics. In 2012 IEEE 13th International Conference on Information Reuse \& Integration (IRI) (pp. 377-384). IEEE.

Wei, W., McCusker, J. H., Hyman, R. W., Jones, T., Ning, Y., Cao, Z., Gu, Z., Brubo, D., Miranda, M., Nguyen, M., Wilhelmy, J., Komp, C., Tamse, R., Wang, X., Jia, P., Luedi, P., Oefner, P. J., David, L., Dietrich, F. S., Li, Y., Davis, R. W., \& Steinmetz, L. M. (2007). Genome sequencing and comparative analysis of Saccharomyces cerevisiae strain YJM789. Proceedings of the National Academy of Sciences, 104(31), 12825-12830.

Yoshimoto, N., Kawai, T., Yoshida, M., \& Izawa, S. (2019). Xylene causes oxidative stress and pronounced translation repression in Saccharomyces cerevisiae. Journal of bioscience and bioengineering, 128(6), 697-703.

Yu, A., Zhou, R., Xia, B., Dang, W., Yang, Z., \& Chen, X. (2020). NAMPT maintains mitochondria content via NRF2PPAR $\alpha / A M P K \alpha$ pathway to promote cell survival under oxidative stress. Cellular Signalling, 66, 109496.

Zhai, Z., Yang, Y., Wang, H., Wang, G., Ren, F., Li, Z., \& Hao, Y. (2020). Global transcriptomic analysis of Lactobacillus plantarum $\mathrm{CAUH} 2$ in response to hydrogen peroxide stress. Food Microbiology, 87, 103389.

Zimmermann, P., Laule, O., Schmitz, J., Hruz, T., Bleuler, S., \& Gruissem, W. (2008). Genevestigator transcriptome meta-analysis and biomarker search using rice and barley gene expression databases. Molecular plant, 1(5), 851-857. 